quibble about minor inaccuracies, and there are more than a few typographical errors.

But all in all, Journey From the Center of the Sun is a fine book. Solar physics is a vibrant field of research, with many spacebased and ground-based observatories either operating or under construction, and many theoretical ideas being fleshed out with high-powered numerical simulations. Zirker has written an entertaining and educational survey of the current state of knowledge of our all-important neighbour that sits at the centre of the Solar System.

James A. Klimchuk is at the Naval Research Laboratory, Washington DC, USA.

\section{Keeping up with evolution}

\section{Encyclopedia of Evolution}

editor-in-chief Mark Pagel

Oxford University Press: 2002. 1,234 pp.

$£ 185, \$ 325$

\section{Joel Peck}

Evolutionary biology is the study of how populations of organisms change over time. Given the relatively small number of working evolutionary biologists, the field receives a surprisingly large amount of media attention. Indeed, evolution is one of the few areas of science that is of genuine interest to a substantial proportion of the public and to first-year undergraduates.

The field has grown enormously in recent years, in part because of progress in research on genomics, physiology, animal behaviour and other related topics. It is difficult to keep up with the key developments in evolutionary research, even for dedicated specialists, so the appearance of the Encyclopedia of Evolution is timely. This two-volume set comprises 365 articles from 330 different authors who cover all of the main areas of evolutionary biology and also explore many minor themes.

The encyclopedia opens with seven 'overview essays', which are written by some of the main contributors to modern evolutionary science, including John Maynard Smith, Jane Goodall and Luigi Luca CavalliSforza. Some of these essays concern obvious organizing themes, such as the major transitions in evolution, macroevolution and the history of evolutionary thought. Others are instructive but rather idiosyncratic choices. The essays include pieces on motherhood, culture in chimpanzees, and darwinian medicine.

The rest of the encyclopedia is taken up with an alphabetical arrangement of short essays, which are generally well written and accessible. Each contains a bibliography, many of which include comments in the form of short annotations. These are very

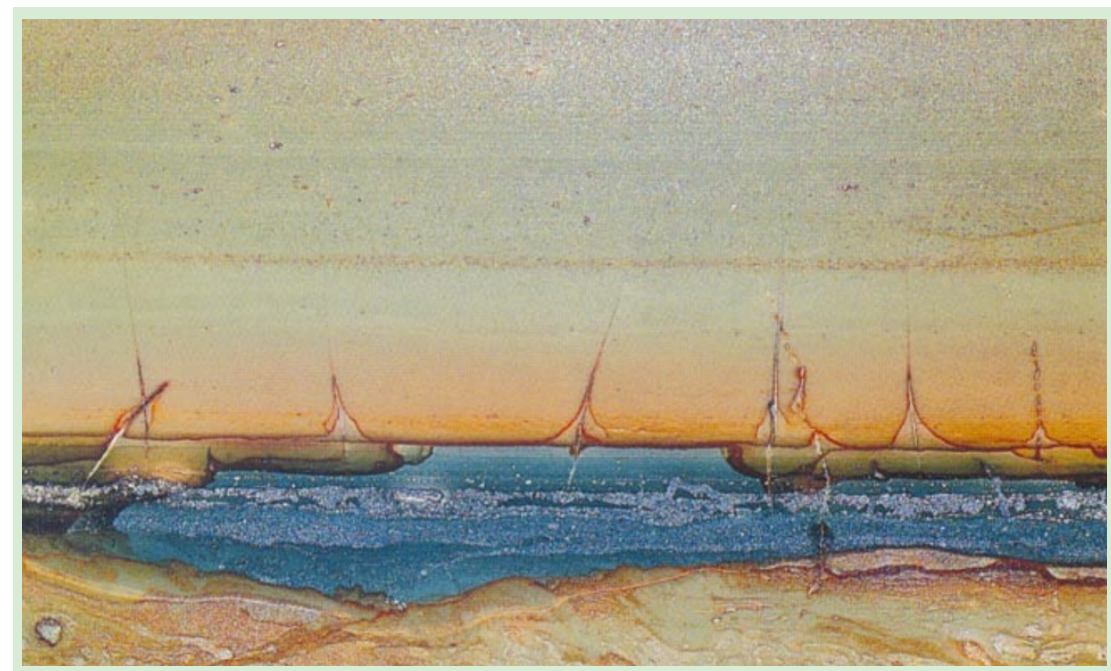

\title{
A natural harbour
}

Do you recognize the harbour in this painting? No, you probably don't, because it's not a painting at all, but the natural colouring on a section of polished paesina stone. This image, along with countless other artistic pictures of minerals and rock formations, can be found in Exposures of Mineral and Rock by Dirk J. Wiersma (Inmerc bv, E49.50). This coffee-table book, which has text in both Dutch and English, is a stunningly illustrated guide to rocks, crystals, geodes, gems, fossils, minerals and all manner of things geological. helpful for anyone trying to navigate an unfamiliar area of the literature. Equations are rare and technical terms are explained. Specialists should not expect to learn much in the way of facts about their own areas, but they may find new perspectives and insights. They will also find articles to bring them up to speed when they explore unfamiliar areas of evolutionary biology.

All of which is not to say that this encyclopedia is a perfect document. For one thing, it is heavy. The two volumes weigh in at more than $2 \mathrm{~kg}$ each, so you will not want to chuck them into your bag very often for a quick read on the bus. Also, I wish that some of the authors could have read drafts of each others' articles. For example, the article on altruism contains a discussion of group selection that doesn't seem to take into account recent advances in the theory underlying this phenomenon, and its list of further reading includes several articles from this encyclopedia, but not the excellent one on group selection!

Another problem is the selection of topics. Most of those that you would expect are here, but there are some important exceptions. There is no article on epistasis, despite the importance of this topic for many of the phenomena that are covered, such as speciation and the evolution of sexual reproduction. The evolution of genetic dominance is also a no-show. However, no encyclopedia can do everything. What is more interesting is the choice of some of the topics that have been included. There are two articles on art, for example, an article on warfare, and even one on globalization. Some of them have only tenuous connections to evolutionary thinking. But more is probably better, and people come to evolutionary studies from many directions. Maybe some of the more far-out articles will help to direct unique and energetic minds towards evolutionary pursuits.

Many of the articles are written by researchers who have made key contributions to the areas about which they are writing. This is good in that the authors are close to the cutting edge of their topics and are able to convey the latest findings. The drawback is that, in some cases, the articles present an enthusiast's view of each topic, rather than the more even-handed account that might be expected had the editors employed professional science writers rather than researchers. Personally, I like the approach taken by the encyclopedia, but readers should recognize that they may have to read material from other sources to obtain a balanced view.

It seems almost certain that this encyclopedia will be a key source for researchers, as well as for students and teachers. Many students will turn to it for help when they start to write an essay or try to find a topic for research. They will find articles that provide a good initial orientation, along with the key references. Teachers will use it for preparing lectures and reading materials, but they will also need a copy to check for cheaters - this is likely to be one of the most widely plagiarized books in the history of evolutionarybiology.

Joel Peck is at the Centre for the Study of

Evolution, University of Sussex, Falmer,

Brighton BN1 9QG, UK. 